\title{
Conditional spatial discrimination in humans with hypoxic brain injury
}

\author{
CATHERINE E. MYERS \\ Rutgers University, Newark, New Jersey \\ RAMONA O. HOPKINS \\ Brigham Young University, Provo, Utah \\ and LDS Hospital, Salt Lake City, Utah \\ RAYMOND P. KESNER \\ University of Utah, Salt Lake City, Utah \\ LAURA MONTI \\ Rush-Presbyterian-St. Luke's Medical Center, Chicago, Illinois \\ and \\ MARK A. GLUCK \\ Rutgers University, Newark, New Jersey
}

\begin{abstract}
Hypoxic brain injury can cause severe anterograde amnesia with intact intelligence and attention in select patients. This neuropsychological profile is similar among a broad class of etiologies resulting in damage to the hippocampus and associated medial temporal structures. Several recent studies have demonstrated that subjects with anterograde amnesia can nonetheless acquire simple associative tasks. In the present study, the performance of subjects with anterograde memory impairments resulting from hypoxic brain injury on a conditional spatial discrimination was examined. Quantitative magnetic resonance imaging revealed significant hippocampal atrophy in the hypoxic subjects. The hypoxic subjects were able to acquire and reverse the discrimination, although they were slower relative to matched control subjects. Hypoxic subjects also showed a tendency to perseverate after reward contingencies were reversed.
\end{abstract}

Hypoxic brain injury can result from a variety of etiologies, including cardiac arrest, respiratory arrest, and carbon monoxide $(\mathrm{CO})$ poisoning. The severity of the injury depends on the length and experience of the hypoxic episode; although nonspecific degenerative neuropathologic changes can ensue, the hippocampus appears to be more selectively vulnerable than other brain structures. For example, several recent studies have presented quantitative magnetic resonance imaging (MRI) analyses showing significant reduction in hippocampal volume in hypoxic subjects (e.g., Hopkins, Gale, et al., 1995; Hopkins, Kesner, \& Goldstein, 1995; Press, Amaral, \& Squire, 1989). Alternatively, hypoxic brain injury may result in diffuse damage throughout the brain in some cases (Bachevalier \& Meunier, 1996; Gale et al., 1999; Hopkins, Gale, et al., 1995). However, in select cases, cell death appears

This research was supported by grants from the Office of Naval Research, through the Young Investigator Program (to M.A.G.) and Grant N00014-88-K-0112 (to M.A.G.), and by a grant from the McDonnellPew Program in Cognitive Neuroscience (to M.A.G. and C.E.M.). Correspondence concerning this article should be addressed to C. E. Myers, Department of Psychology, Rutgers University, Newark, NJ 07102 (email: myers@pavlov.rutgers.edu). limited to (or particularly severe in) the hippocampus (e.g., Hopkins, Gale, et al., 1995; Hopkins, Kesner, \& Goldstein, 1995; Press et al., 1989; Zola-Morgan, Squire, \& Amaral, 1986).

Consistent with these anatomical findings is research showing that hypoxic subjects may exhibit severe anterograde amnesia in the absence of impaired intelligence and attention (Hopkins, Kesner, \& Goldstein, 1995; Press et al., 1989; Zola-Morgan et al., 1986). These findings are similar to the anterograde amnesia syndrome exhibited by individuals who sustain damage to the hippocampus and related structures through stroke or other insult to the medial temporal lobes (e.g., Zola-Morgan \& Squire, 1990). For these reasons, hypoxic brain injury is often grouped within the broader class of etiologies resulting in anterograde amnesia because of damage to the hippocampus and related medial temporal lobe structures.

A small but growing literature is demonstrating that, although subjects with anterograde amnesia are profoundly impaired at acquiring new memories, they may still be able to demonstrate associative learning at normal or nearnormal speed. For example, amnesic subjects have been shown able to acquire classically conditioned motor reflex responses (Gabrieli et al., 1995), cognitive and motor 
Table 1

Demographic Data for Hypoxic and Control Subjects

\begin{tabular}{clll}
\hline \multicolumn{1}{c}{ ID } & Etiology & Sex & Age \\
\hline Hypoxic subjects & & & \\
H-1 & ARDS & M & 72 \\
H-2 & ARDS & F & 74 \\
H-3 & ARDS & M & 19 \\
H-4 & C/RA & M & 32 \\
H-5 & CO & F & 43 \\
H-6 & C/RA & M & 37 \\
Control subjects & & & \\
C-1 & & M & 62 \\
C-2 & & F & 75 \\
C-3 & & M & 19 \\
C-4 & & M & 32 \\
C-5 & & F & 43 \\
C-6 & & M & 35 \\
\hline
\end{tabular}

Note--ARDS, acute respiratory distress syndrome; C/RA, cardiac and/or respiratory arrest; $\mathrm{CO}$, carbon monoxide poisoning.

skills (Cohen, 1984), auditory and visual emotional conditioning (Bechara et al., 1995), and probabilistic category rules (Knowlton, Squire, \& Gluck, 1994). As these associative tasks become more complex, amnesic subjects often demonstrate impairments. One example is conditional spatial discrimination, in which subjects are rewarded for a left or a right response, depending on which of two cues is present. This kind of task is often disrupted in animals with broad lesions of the hippocampus and associated structures (see, e.g., Kimble, 1963; Marston, Everitt, \& Robbins, 1993).

Daum, Schugens, Channon, Polkey, and Gray (1991) recently considered conditional spatial discrimination in human subjects with unilateral temporal lobe resection. Subjects saw an animated mouse running through a Tmaze and had to guide the mouse into the left or right maze arm to obtain a reward; the correct choice was determined by the screen background color. Daum et al. found that subjects with right temporal lobe (RTL) resection were impaired at acquiring the response rule, but not at reversing it. The same study found no acquisition or reversal deficits in subjects with left temporal lobe (LTL) removal and only a mild reversal deficit in subjects with frontal lobe damage. Since the temporal lobe resections included hippocampal removal, Daum et al. concluded that the right hippocampus and associated nearby structures were critical for acquiring the conditional spatial discrimination. However, the RTL subjects in the Daum et al. study do not appear to have been densely amnesic; for example, Daum et al. report that, although about half of the LTL subjects were impaired on the verbal paired associate subtest of the Weschler Memory Scale (WMS), the RTL subjects performed within the normal range.

The purpose of the present study was to examine a population of hypoxic subjects with anterograde amnesia on a conditional spatial discrimination modeled after the Daum et al. (1991) paradigm. Subjects were selected from a population that had experienced hypoxic brain injury re- sulting in severe memory deficits and hippocampal damage. The aim of the study was to address several basic questions. First, could this amnesic population acquire a conditional spatial discrimination? If so, would hypoxic subjects acquire the conditional spatial discrimination at the same speed as matched control subjects? Would hypoxic subjects be able to reverse this discrimination, and at what speed? And finally, apart from overall speed of learning, would hypoxic subjects show any qualitative differences from matched controls in the pattern of errors?

\section{METHOD}

\section{Subjects}

Six individuals who had experienced a hypoxic episode were recruited for this study. The etiologies of the hypoxic subjects included cardiac/respiratory arrest, $\mathrm{CO}$ poisoning, and acute respiratory distress syndrome (ARDS). These subjects were recruited through the LDS Hospital Carbon Monoxide and ARDS Studies program in Salt Lake City, except for Subject H6 who was recruited and tested at Rush-Presbyterian-St. Luke's Hospital in Chicago. The demographic data are summarized in Table 1.

Six age- and gender-matched control subjects were recruited through local advertising in Salt Lake City and Newark. There were 4 males and 2 females in each group. The mean age of the hypoxic subjects was 46.2 years $(S E=9.1)$, and the mean age of the control subjects was 46.3 years $(S E=8.6)$. There was no significant difference for age between the two groups [ $t$ test: $t(10)=0.15, p=.88$ ] The mean education level of the hypoxic group was $12.0 \pm 0$ years and the mean education level of the control group was 13.0 1.2 years. There was no significant difference for education level between the two groups [ $t$ test: $t(10)=1.93, p=.08$ ]

Informed consent was obtained for all the subjects. No control subject had a prior history of neurologic disorder, alcohol or drug abuse, or psychiatric disturbance.

\section{Neuropsychological Testing}

The hypoxic subjects were tested approximately 1 year after the hypoxic event. Hypoxic subjects were administered a battery of neuropsychological tests to assess memory, intelligence, and attention.

To assess memory, subjects were administered the revised WMS (WMS-R). This test generates a variety of subscores, including a General Memory Index (GMI), a Delayed Recall Index, and an Attention/Concentration Index. These scores are age adjusted, with normal performance defined as $100 \pm 15$. On the WMS-R GMI the mean score was 85.3 $(S E=7.3)$, and for the Attention/Concentration Index the mean score was $101.3(S E=9.3)$. As is shown in Table 2, the hypoxic subjects generally exhibited poor memory performance, particularly on the Delayed Recall Index: All except $\mathrm{H} 1$ and $\mathrm{H} 2$ scored more than one standard deviation below normal. Two subjects received the lowest possible score of 50 on the Delayed Recall Index.

In addition, the subjects were administered the Rey-Osterrieth Complex Figure Test (complex figure), which involves copying a complex geometric figure (36 points possible) followed, after $30 \mathrm{~min}$, by a recall test in which subjects are asked to draw the figure from memory. The hypoxic subjects were slightly impaired on the copy portion and dramatically impaired on the recall portion. Table 2 shows the copy and delay recall scores, as well as the differences between these scores and age-adjusted norms (Spreen \& Strauss, 1991). All hypoxic subjects except H6 scored well below age-appropriate means. Two hypoxic subjects ( $\mathrm{H} 1$ and $\mathrm{H} 2)$ scored within the normal range on the WMS-R Delayed Recall Index. However, on the copy of a complex figure, these two individuals 
Table 2

Neuropsychology Test Results for Hypoxic Subjects, With Sample Means and Standard Deviations

\begin{tabular}{|c|c|c|c|c|c|c|c|}
\hline \multirow[b]{2}{*}{ Subject } & \multicolumn{3}{|c|}{ WMS-R } & \multirow{2}{*}{$\begin{array}{l}\text { WAIS-R } \\
\text { (FSIQ) }\end{array}$} & \multicolumn{3}{|c|}{ Rey-Osterrieth } \\
\hline & GMI & Delay & Attn & & Copy & Delay & Diff \\
\hline $\mathrm{H} 1$ & 97 & 89 & 116 & 102 & 21 & 2 & -9.8 \\
\hline $\mathrm{H} 2$ & 113 & 104 & 97 & 109 & 19 & 5 & -6.8 \\
\hline H3 & 65 & 50 & 96 & 95 & 32 & 10 & -9.5 \\
\hline H4 & 70 & 50 & 87 & 90 & 32 & 5 & -14.5 \\
\hline H5 & 89 & 80 & 88 & 100 & 36 & 26 & +6.5 \\
\hline H6 & 81 & 57 & 124 & 99 & n.a. & n.a. & n.a. \\
\hline$M$ & 85.8 & 71.7 & 101.3 & 99.2 & 28.0 & 9.6 & -6.8 \\
\hline$S D$ & 17.8 & 22.7 & 15.2 & 6.4 & 7.5 & 9.6 & 7.9 \\
\hline
\end{tabular}

Note-WMS-R, Weschler Memory Scale-Revised; WAIS-R, Weschler Adult Intelligence Scale-Revised; GMI, General Memory Index; Delay, Delayed Recall Index; Attn, Attention/Concentration Index; FSIQ, Full-Scale Intelligence Quotient; n.a., not available. The Rey-Osterrieth Diff score is the difference between the mean Delay score and the age-appropriate norm.

scored 21 and 19 , but only 2.0 and 5.0 on the delayed recall of the complex figure. All the hypoxic subjects were significantly impaired on at least one of the two memory measures.

Hypoxic subjects were administered the Weschler Adult Intelligence Scale-Revised (WAIS-R), to assess intelligence. This test generates age-adjusted scores, including the Full-Scale Intelligence Quotient (FSIQ), with normal performance defined as $100 \pm 15$. For the 6 hypoxic subjects, the mean FSIQ score was 99.7 ( $S E=2.7$ ), the mean Verbal Intelligence Quotient was $101.2(S E=3.4)$, and the mean Performance Intelligence Quotient was $98.2(S E=4.7)$. Table 2 shows that for all of the hypoxic subjects, FSIQ scores were within the normal range.

Finally, the Attention/Concentration Index of the WMS-R, a measure of attention, was administered, with age-adjusted scores of $100 \pm 15$ considered normal. Hypoxic subjects' performance was within the normal range. On the basis of these tests, all the hypoxic subjects exhibited impaired memory, based on the WMS-R Delayed Recall Index and/or the Rey-Osterrieth delay recall scores, but their performance on the WAIS-R FSIQ and the WMS-R Attention/Concentration Index was within the normal range.

\section{Neuroimaging}

Magnetic resonance imaging. MR images were acquired at 1.5 Tesla with a quadriture head coil, using standard clinical protocols. Sagittal T1-weighted (500/11/2; TR/TE/excitations) images were first acquired, followed by axial and coronal proton density and T2weighted $(3000 / 31 ; 90 / 1)$ spin echo images. For the axial images, the slice thickness was $5 \mathrm{~mm}$, with a $2-\mathrm{mm}$ interslice space. Images were acquired on a $256 \times 192$ matrix with a $22-\mathrm{cm}$ field of view for the axial images and a $24-\mathrm{cm}$ field of view for the sagittal images. Coronal images were 3-mm-thick interleaved slices acquired on a $512 \times 256$ matrix with a $22-\mathrm{cm}$ FOV. Flow compensation, an inferior saturation pulse, and variable bandwidth were used.

Quantitative magnetic resonance imaging. Quantitative, or volumetric, analysis of cerebral structures obtained from MRI (QMRI) were performed on all patients as per the methods described previously (using the software ANALYZE; Bigler et al., 1997; Biomedical Imaging Resource, 1993; Blatter et al., 1995). Hippocampal volumetric measures were obtained in 4 of the 6 subjects. We were unable to obtain MR images in 2 subjects, since 1 subject lived in Chicago and the other refused MR imaging. We used a normative database that allowed comparisons with specific ageand gender-matched control groups, as described by Bigler et al. Hippocampal volume was considered abnormal if it was more than one standard deviation below the mean based on this normative sample.

\section{Procedure: Conditional Spatial Discrimination}

The conditional spatial discrimination task was an adaptation of that described by Daum et al. (1991) and took the form of a computerized game in which the subject viewed an animated mouse running in a T-maze. The game was implemented on Macintosh LC and PowerBook series computers, programmed in the SuperCard language. The keyboard was masked, except for two adjacent keys, labeled "left" and "right," which were used to record the subject's responses. The subjects were seated in a quiet room and shown the following instructions on a computer screen: "You are a hungry mouse in a maze. Direct your mouse left or right to eat as much cheese as possible. Press the button to begin." The experimenter read these instructions aloud to the subject, demonstrated the "left" and "right" response keys, and pressed the computer mouse button to begin the experiment.

On each trial, a T-maze was displayed with a black square at the end of each arm serving as a visual blocker. The mouse appeared in the start box at the bottom of the screen (Figure $1 \mathrm{~A}$ ) and ran to a choice point in the center of the maze (Figure 1B). The mouse hesitated there, wavering back and forth, until the subject pressed a key to send it into either the left or the right maze arm. If the subject chose correctly (Figure IC), when the mouse reached the end of the maze arm, the blocker was removed to reveal cheese, and a "happy" mouse sound was played through the computer speakers. If the subject chose incorrectly (Figure ID), when the mouse reached the end of the maze arm, the blocker was removed to reveal no cheese, and a "sad" mouse sound was played through the computer speakers.

On each trial, cheese was present in either the left or the right maze arm; its placement depended on the background screen brightness. For all the subjects in the present experiment, cheese was located in the right-hand arm if the background was light gray and in the left-hand arm if the background was dark gray. This mapping was held consistent across subjects because of the small number of subjects in the present study; in their earlier study, Daum et al. (1991) counterbalanced mapping and found no significant effects of this mapping. All the subjects received the same trial order, which was a pseudorandom series of trials constrained so that the same background never occurred more than three times in a row.

This initial acquisition phase lasted until the subject made eight consecutive correct responses or to a maximum of 90 trials. At this point, the first reversal occurred: The color-cheese mapping was reversed so that cheese was in the left-hand arm for a light-gray background and in the right-hand arm for a dark-gray background The subject was given no indication that a reversal had occurred. This first reversal phase continued until the subject made eight consecutive correct responses or to a maximum of 50 trials. 
(A) Start of Trial

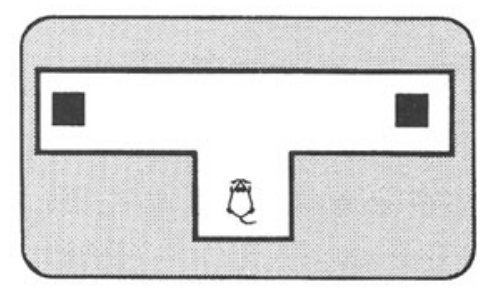

(C) Correct Response

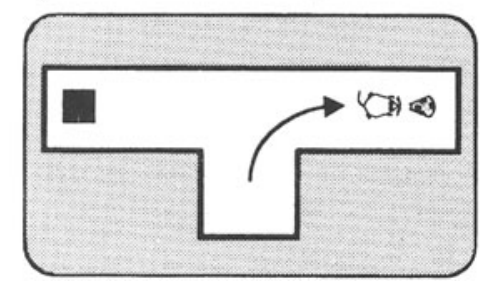

(B) During Trial

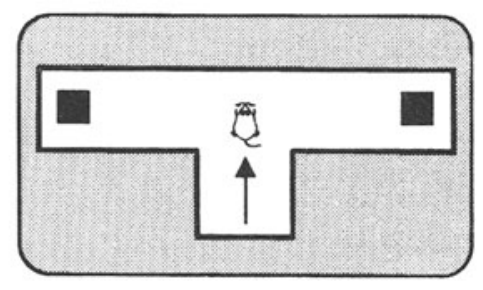

(D) Incorrect Response

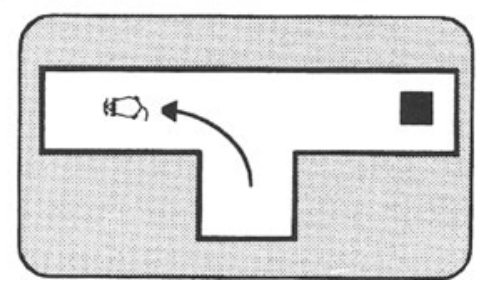

Figure 1. Screen events during an experimental trial. At the start of a trial, the animated mouse appears in the start box $(A)$, runs to the choice point $(B)$, and wavers there until the subject responds by pressing the "left" or "right" key. The mouse then runs to the chosen maze arm. If this choice was correct, cheese is revealed, and a "happy" mouse sound is played (C). If the choice is incorrect, there is no cheese, and a "sad" sound is played (D).

Elaborating on the original paradigm of Daum et al. (1991), we added a second and third reversal phase. These reversals were also unsignaled, and each lasted until the criterion of eight consecutive correct responses was reached or to a maximum of 50 trials.

After the third reversal was completed, the subjects saw a message informing them how many pieces of cheese they had found during the experiment and thanking them for participating. The total experiment took approximately $10-15 \mathrm{~min}$ to complete, depending on how quickly the subject reached criterion in each phase.

\section{Data Collection}

The computer recorded individual responses and target responses for each trial. The computer terminated a phase when the subject achieved eight consecutive correct responses or when the maximum number of trials was exhausted. For each subject, the total trials to criterion (including the final eight consecutive correct) was recorded for each phase. Since each reversal was unsignaled to the subject, it was expected that each subject should respond incorrectly on the first trial following the reversal; optimally, a subject might then immediately change response rules and proceed to make eight consecutive responses. Such a one-trial reversal would result in a score of nine for the reversal phase.

Alternatively, a subject might require several trials before successfully reversing the response rule. Several authors have noted that reversal typically involves three distinct phases (e.g., Eichenbaum, Fagan, \& Cohen, 1986; Jones \& Mishkin, 1972). The phases include a perseveration phase, defined as a string of incorrect responses before the old response rule is abandoned, a phase of mixed correct and incorrect responses while new response rules are evaluated, and finally, a correct phase leading to criterion performance. Here, the perseveration phase is of particular interest, since difficulty abandoning previously reinforced strategies is noted in several patient populations, especially those with frontal damage (e.g., Lezak, 1995). Accordingly, the computer calculated a perseveration score for each subject for each reversal, defined as the number of consecutive incorrect responses following a reversal, excluding the first (obligatory) incorrect response.

\section{RESULTS}

\section{Magnetic Resonance Imaging Results}

All 4 of the hypoxic patients with QMRI had significant hippocampal atrophy, since the right and left hippocampal volumes were at least one standard deviation below the normal control group. Clinical brain MRI reports by the neuroradiologist, for the 4 hypoxic subjects, indicated no evidence of lesions or other structural abnormalities. Table 3 shows the volumetric hippocampal data for the hypoxic subjects. The mean number of standard deviations from age-matched normal control subjects for the hypoxic subjects' right hippocampus was $-2.83 \pm 1.7(S E=0.87)$, and for the left hippocampus, it was $-2.01 \pm 1.5(S E=0.75)$. Figure 2 shows coronal sections through the hippocampus of a hypoxic subject $(\mathrm{H}-4)$ and an age-matched normal individual.

\section{Conditional Spatial Discrimination}

All the subjects acquired the original conditional discrimination within the maximum 90 trials. Figure 3A shows the total trials to criterion on the acquisition phase. The hypoxic subjects averaged 39.7 trials to criterion $(S E=10.5)$, whereas the control subjects averaged only 22.0 trials $(S E=7.2)$. This difference failed to reach statistical significance (Mann-Whitney $U=26.0, p>.05$ ). Figure 3B shows the total errors on the acquisition phase. 
Table 3

Quantitative Magnetic Resonance Imaging (MRI)

Data for Hypoxic and Normative Subjects

\begin{tabular}{|c|c|c|c|c|c|}
\hline \multirow{2}{*}{\multicolumn{3}{|c|}{$\begin{array}{l}\text { Hypoxic Subjects: } \\
\text { Hippocampal Volume in } \mathrm{cc}^{3}\end{array}$}} & \multicolumn{3}{|c|}{$\begin{array}{c}\text { Control Subjects: } \\
\text { Hippocampal Volume in } \mathrm{cc}^{3}\end{array}$} \\
\hline & & & \multirow{2}{*}{$\begin{array}{c}\text { Mean } \pm S D \\
\text { Right }\end{array}$} & \multirow{2}{*}{$\begin{array}{c}\text { Mean } \pm S D \\
\text { Left }\end{array}$} & \multirow{2}{*}{$\begin{array}{c}\text { Number of } S D s \\
\text { Below Normals } \\
\text { Right/Left }\end{array}$} \\
\hline ID & Right & Left & & & \\
\hline $\begin{array}{l}\mathrm{H}-1 \\
\mathrm{H}-2\end{array}$ & $\begin{array}{l}1.82 \\
\text { no MRI }\end{array}$ & 2.06 & $2.55 \pm 0.28$ & $2.58 \pm 0.24$ & $-3.5 /-1.81$ \\
\hline $\mathrm{H}-3$ & 2.19 & 2.34 & $2.63 \pm 0.28$ & $2.68 \pm 0.25$ & $-1.6 /-1.4$ \\
\hline $\mathrm{H}-4$ & 1.41 & 1.29 & $2.77 \pm 0.27$ & $2.64 \pm 0.32$ & $-5.0 /-4.2$ \\
\hline $\begin{array}{l}\mathrm{H}-5 \\
\mathrm{H}-6\end{array}$ & $\begin{array}{l}2.42 \\
\text { no MRI }\end{array}$ & 2.38 & $2.55 \pm 0.28$ & $2.58 \pm 0.24$ & $-1.5 /-1.06$ \\
\hline
\end{tabular}

Note-The normative data from age-matched normal control subjects were taken from Bigler et al. (1997).

Again, the hypoxic subjects averaged more errors than did the control subjects, but this difference failed to reach statistical significance on an analysis of variance [ANOVA; $F(1,10)=1.85, p>.05]$. Thus, the hypoxic subjects were not impaired, relative to control subjects, in acquiring the conditional spatial discrimination.

The control subjects reversed the discrimination quickly, reaching criterion within an average of 10.3 trials $(S E=1.1)$. Four control subjects made optimal onetrial reversals. By contrast, the hypoxic subjects averaged 15.8 trials to reverse $(S E=1.4)$, and no hypoxic subjects made one-trial reversals. The difference between hypoxic and control groups was significant $[F(1,10)=9.44, p=$ $.012]$; thus, the control subjects were faster to reverse the discrimination than were the hypoxic subjects.
All the subjects completed all reversals within the maximum allowable trials. Figure 4 shows the average errors on each of the three successive reversals for the control and hypoxic subjects. Over all three successive reversals, the hypoxic subjects made significantly more errors than the control subjects did [repeated measures ANOVA, $F(1,10)=5.33, p=.04]$. By the third reversal, all the control subjects made at least two optimal one-trial reversals, whereas only 1 hypoxic subject (H-6) made two one-trial reversals and 2 hypoxic subjects ( $\mathrm{H}-2$ and $\mathrm{H}-4)$ never made a one-trial reversal.

Figure 5 shows the mean number of perseverative responses for each reversal, defined as the string of consecutive incorrect responses immediately following a reversal, excluding the first, obligatory incorrect response.
(A)

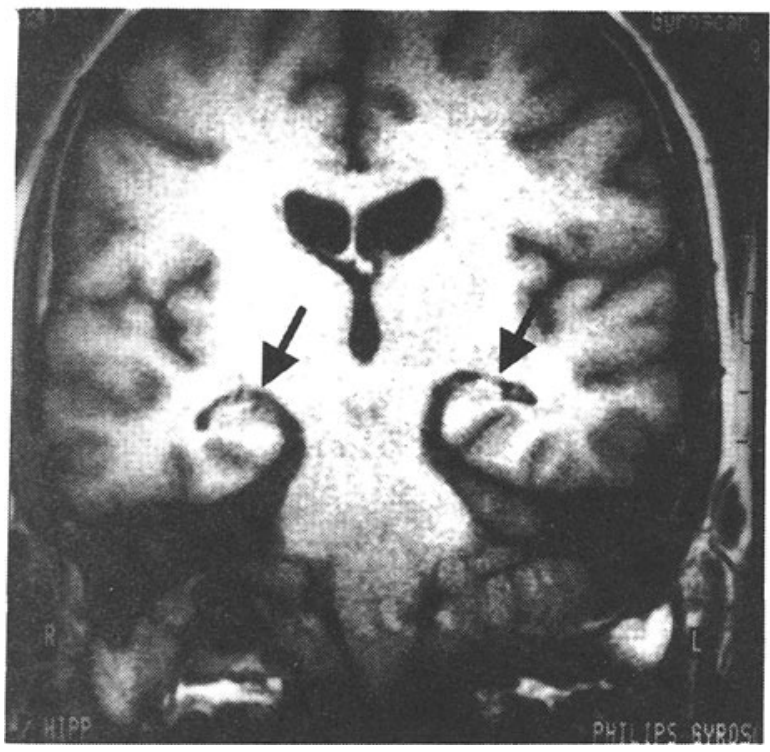

(B)

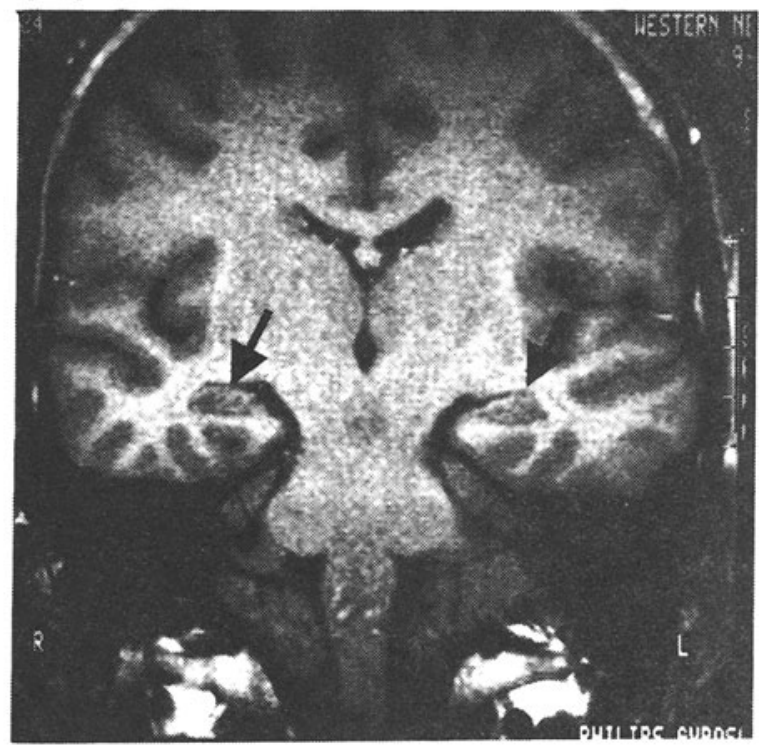

Figure 2. Magnetic resonance images of coronal sections through the hippocampus in a hypoxic and a control subject. The hypoxic subject (panel A) showing bilateral atrophy of the hippocampus (arrows) is compared with the normal subject in panel B. The subject shown in panel $A$ is hypoxic subject $H-4$ of the present study. 

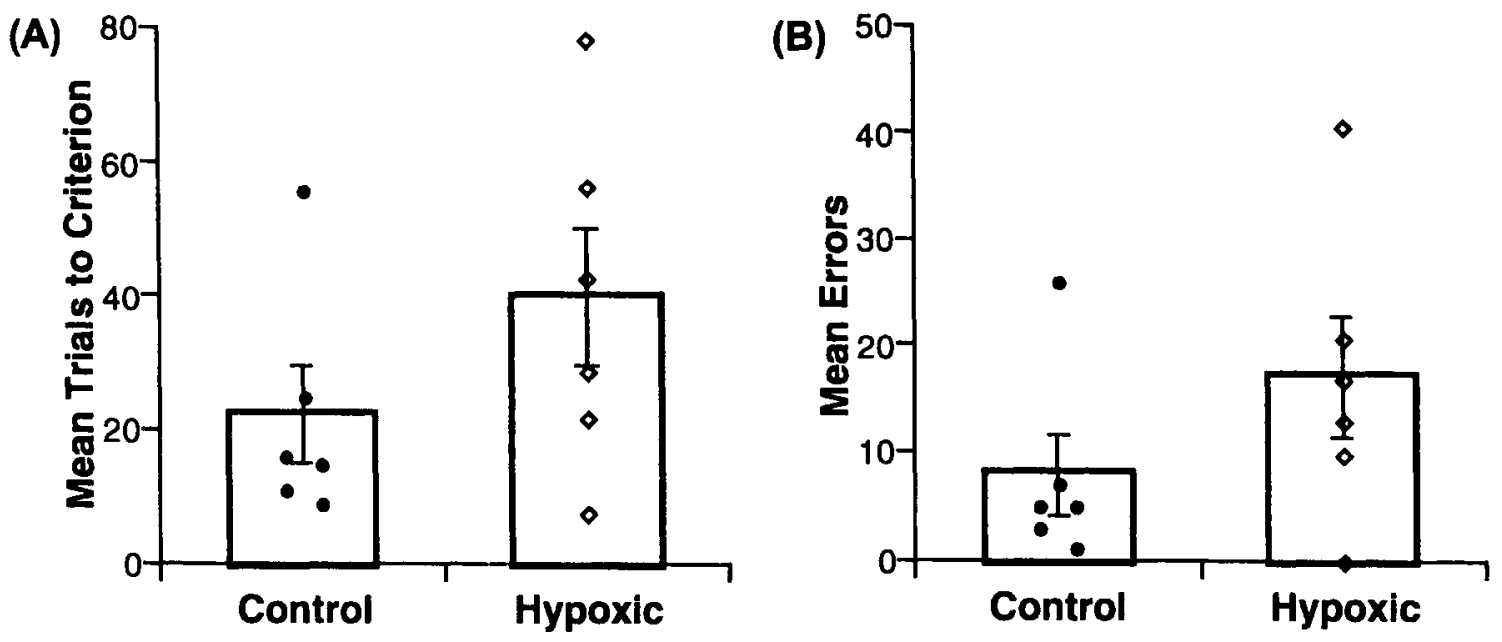

Figure 3. The acquisition phase of the conditional spatial discrimination: (A) mean trials to criterion; (B) mean total errors. Circles and diamonds show individual subject performance.

The control subjects made virtually no perseverative responses, tending to abandon the old response strategy as soon as it ceased to be rewarded. The hypoxic subjects, on the other hand, showed more perseverative errors, a difference that, although small in magnitude, was highly significant [repeated measures ANOVA, $F(1,10)=6.54$, $p=.028]$. There was no within-subjects effect of repeated reversal $[F(2,20)=1.46, p=.26]$.

\section{DISCUSSION}

The first finding of this study was that subjects with anterograde amnesia resulting from hypoxic brain injury could learn and reverse a conditional spatial discrimination. This adds to a small but growing literature docu- menting that humans with anterograde amnesia can acquire simple categorization tasks (e.g., Knowlton, Ramus, \& Squire, 1992; Knowlton et al., 1994).

The hypoxic subjects did take a somewhat greater number of trials than did the control subjects to reach criterion on the original discrimination, although this difference fell short of statistical significance. On average, the hypoxic subjects required 39.7 trials to acquire the discrimination. In their study, Daum et al. (1991) reported that subjects with right temporal lobectomy acquired the same discrimination with a median of about 37 trials. Daum et al.'s control subjects mastered the acquisition with a median of just over 20 trials, which is again comparable with the mean of 22 trials in our control subjects. Thus, although the difference between the hypoxic and

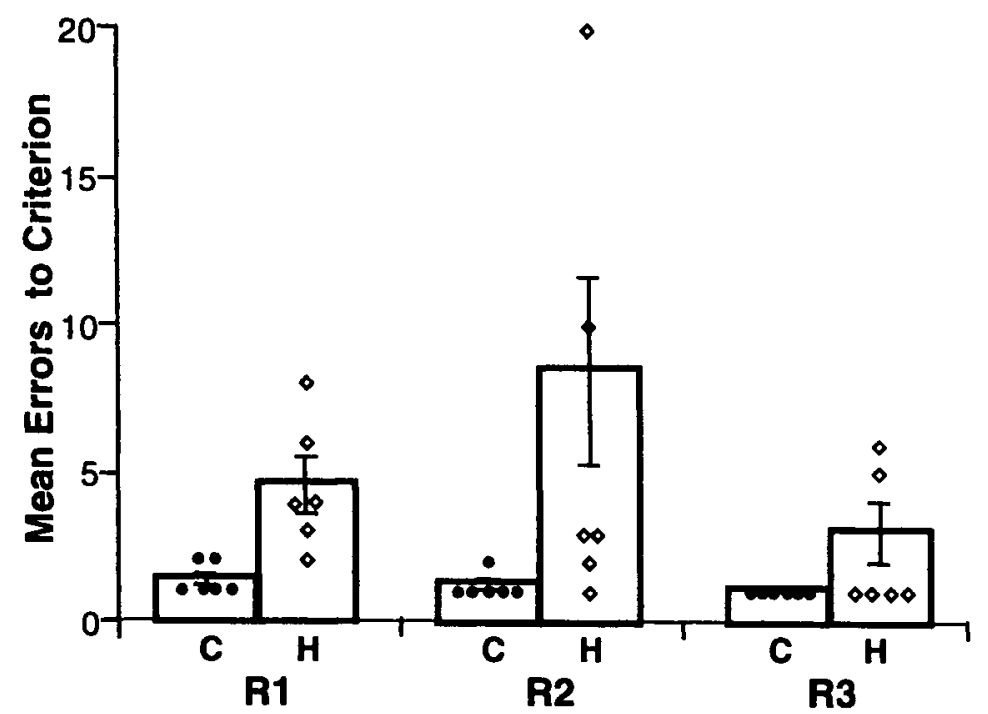

Figure 4. Mean trials to criterion performance on the three successive reversals, R1-R3. 


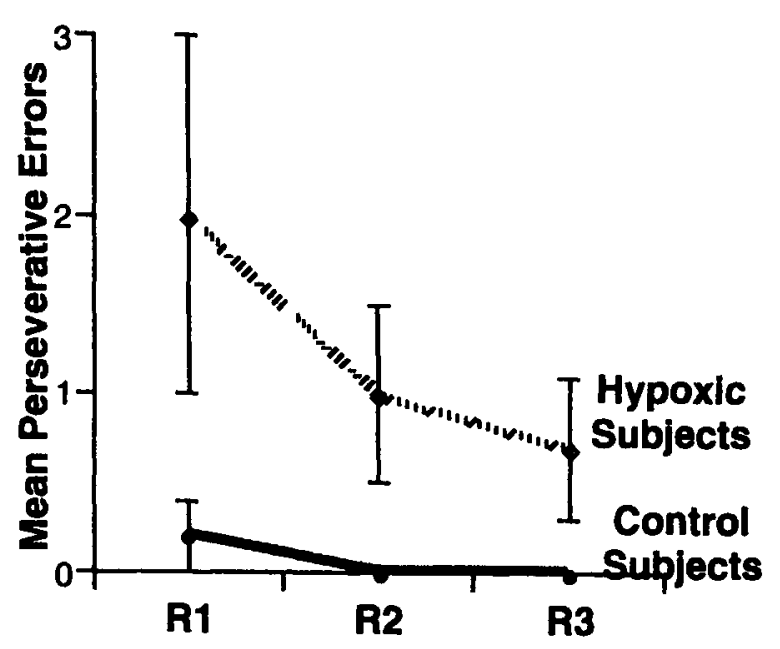

Figure 5. Mean perseverative errors, defined as consecutive errors following a reversal (excluding the first, obligatory error), for each reversal phase $R 1-R 3$.

the control subjects in the present study fell short of statistical significance, it is quite consonant with the results of Daum et al., who found impaired acquisition following right temporal lobectomy.

Hypoxic brain injury may result in bilateral damage to the hippocampus and related medial temporal lobe structures in select subjects (Hopkins, Gale, et al., 1995; Hopkins, Kesner, \& Goldstein, 1995; Press et al., 1989; Squire \& Zola, 1996). In fact, 4 of our 6 subjects had documented bilateral medial temporal lobe damage. The remaining 2 subjects had etiologies and neuropsychological profiles that would be consisted with presumed bilateral medial temporal lobe damage; their performance on the conditional spatial discrimination was not obviously different from that of the subjects for whom imaging was available. Thus, it is possible that the present results reflect involvement of medial temporal structures in conditioned spatial discrimination. This would be consistent with a recent study showing that ischaemic damage resulting in selective lesions of hippocampal subfield CAl can suffice to cause a strong impairment in a conditional visuospatial task similar to that considered here but spare several other simpler visual and spatial discriminations (Ridley, Timothy, Maclean, \& Baker, 1995).

Daum et al. (1991) also found that their unilaterally lesioned subjects with right temporal lobectomy were not impaired at reversing the conditional spatial discrimination. This contrasts with the hypoxic subjects in the present study, who showed significant reversal impairments. Whereas about $50 \%$ of Daum et al.'s subjects with right temporal lobectomy showed one-trial reversal, none of the hypoxic subjects in the present study showed one-trial reversal on the first reversal phase. The hypoxic subjects continued to show impairments even after multiple successive reversals. The control subjects, by contrast, performed close to the optimum on all three reversals. In ani- mals, medial temporal damage has been shown sometimes to impair reversal of a conditioned spatial discrimination (e.g., Fagan \& Olton, 1986; Silveira \& Kimble, 1968) and has sometimes been shown to have no effect (e.g., Marston et al., 1993; Zola \& Mahut, 1973). Hippocampal region damage has also been shown sometimes to impair nonspatial reversal learning (e.g., Berger \& Orr, 1983). The data from the present study suggest that human amnesic subjects are impaired at reversal; further studies are again indicated to determine whether individuals with bilateral medial temporal damage from other etiologies reliably show similar impairments.

One important finding in this study is that the hypoxic subjects showed significantly more perseverative errors than did the matched controls, continuing to apply a previous response rule even after the reversal occurred. Daum et al. (1991) reported no such perseverative deficits in the subjects with (right or left) unilateral temporal lobe resection; however, there was a slight tendency to perseverate noted among subjects with frontal resection. Thus, one interpretation of the present results is that the hypoxic group did indeed have collateral frontal damage that increased perseveration. However, this is unlikely, given the hypoxic subjects' clinical and quantitative MRI data. A second possibility is that hippocampal damage itself contributes to increased perseveration in some individuals, consistent with various descriptions of hippocampallesioned animals as being unable to inhibit previously reinforced strategies (e.g., Douglas, 1967; Kimble, 1968).

One difficulty in evaluating subjects with hypoxic brain injury is that, although hippocampal region damage may be dramatic and visible (Figure 2), there may be more diffuse damage that is not so easily assessed but may, nonetheless, impact performance (Bachevalier \& Meunier, 1996; Hopkins, Gale, et al., 1995). The clinical MR and QMRI data from our hypoxic subjects support the idea that hypoxia may result in selective damage of the hippocampus, with relative sparing of the frontal lobes and visual association cortex. However, it is possible that functional imaging techniques, such as positron emission tomography and single photon computed tomography, may demonstrate more widespread damage to other neural structures. Further studies are obviously indicated to explore this issue more fully.

\section{REFERENCES}

Bachevalier, J., \& Meunier, M. (1996). Cerebral ischemia: Are the memory deficits associated with hippocampal cell loss? Hippocampus, 6, 553-560.

Bechara, A., Tranel, D., Damasio, H., Adolphs, R., Rockland, C., \& DAMASIO, A. R. (1995). Double dissociation of conditioning and declarative knowledge relative to the amygdala and hippocampus in humans. Science, 296, 115-118

Berger, T. W., \& ORR, W. B. (1983). Hippocampectomy selectively disrupts discrimination reversal conditioning of the rabbit nictitating membrane response. Behavioural Brain Research, 8, 49-68.

Bigler, E., Blatter, D., Anderson, C., Johnson, S., Gale, S., HopKINS, R., \& BURNETT, B. (1997). Hippocampal volume in normal aging and traumatic brain injury. American Journal of Neuroradiology, 18, $11-23$ 
Biomedical Imaging Resource (1993). ANALYZE 6.0 [Computer software]. Rochester, MN: Mayo Foundation.

Blatter, D., Bigler, E., Gale, S., Johnson, S., Anderson, C., BurNETT, B., Parker, N., KurTh, S., \& HorN, S. (1995). Quantitative volumetric analysis of brain MRI: Normative database spanning five decades of life. American Journal of Neuroradiology, 16, 241-251.

CoHen, N. (1984). Preserved learning capacity in amnesia: Evidence for multiple learning systems. In L. R. Squire \& N. Butters (Eds.), Neuropsychology of memory (pp. 83-103). New York: Guilford.

Daum, I., Schugens, M., Channon, S., Polkey, C., \& Gray, J. (1991). T-Maze discrimination and reversal learning after unilateral temporal or frontal lobe lesions in man. Cortex, 27, 613-622.

Dovglas, R. J. (1967). The hippocampus and behavior. Psychological Bulletin, 67, 416-442.

Eichenbaum, H., FAgan, A., \& CoHEn, N. J. (1986). Normal olfactory discrimination learning set and facilitation of reversal learning after medial-temporal damage in rats: Implication for an account of preserved learning abilities in amnesia. Journal of Neuroscience, $\mathbf{6}$, 1876-1884.

FAGAN, A., \& OLTON, D. (1986). Learning sets, discrimination reversal, and hippocampal function. Behavioural Brain Research, 21, 13-20.

Gabrieli, J., McGlinchey-Berroth, R., Carrillo, M., Gluck, M., CermaCK, L., \& Disterhoft, J. (1995). Intact delay-eyeblink classical conditioning in amnesia. Behavioral Neuroscience, 109, 819-827.

Gale, S., Hopkins, R., Weaver, L., Bigler, E., Booth, E., \& BlatTER, D. (1999). MRI, Quantitative MRI, SPECT, and neuropsychological findings following carbon monoxide poisoning. Brain Injury, 13, 229-243.

Hopkins, R., Gale, S., Johnson, S., Anderson, C., Bigler, E., BlatTER, D., \& WEAVER, L. (1995). Severe anoxia with and without concomitant brain atrophy and neuropsychological impairments. Journal of the International Neuropsychological Society, 1, 501-509.

Hopkins, R., KESNER, R., \& GoldSTEIN, M. (1995). Item and order recognition memory in subjects with hypoxic brain injury. Brain \& Cognition, 27, 180-201.

Jones, B., \& Mishin, M. (1972). Limbic lesions and the problem of stimulus-reinforcement association. Experimental Neurology, 36, 362-377.

Kimble, D. P. (1963). The effects of bilateral hippocampal lesions in rats. Journal of Comparative \& Physiological Psychology, 56, 273283.

Kimble, D. P. (1968). Hippocampus and internal inhibition. Psychological Bulletin, 70, 285-295.
KNOWLton, B., Ramus, S., \& SQUiRe, L. (1992). Intact artificial grammar learning in amnesia: Dissociation of classification learning and explicit memory for specific instances. Psychological Science, 3, 172 179.

Knowlton, B., Squire, L., \& Gluck, M. (1994). Probabilistic classification learning in amnesia. Learning \& Memory, 1, 106-120.

LeZAK, M. (1995). Neuropsychological assessment (3rd ed.). New York: Oxford University Press.

Marston, H., Everitt, B., \& Robbins, T. (1993). Comparative effects of excitotoxic lesions of the hippocampus and septum/diagonal band on conditional visual discrimination and spatial learning. Neuropsychologia, 31, 1099-1118.

Press, G., Amaral, D., \& SQuire, L. (1989). Hippocampal abnormalities in amnesic patients revealed by high-resolution magnetic resonance imaging. Nature, 341, 54-57.

Ridley, R., Timothy, C., Maclean, C., \& Baker, H. (1995). Conditional learning and memory impairments following neurotoxic lesion of the CA1 field in hippocampus. Neuroscience, 67, 263-275.

SilveirA, J., \& KimBle, D. (1968). Brightness discrimination and reversal in hippocampally-lesioned rats. Physiology \& Behavior, 3, 625 630.

SPREEN, O., \& Strauss, E. (1991). A compendium of neuropsychological tests: Administration, norms and commentary. New York: Oxford University Press.

SQuire, L., \& ZolA, S. (1996). Ischemic brain damage and memory impairment: A commentary. Hippocampus, 6, 546-552.

Zola, S., \& Mahut, H. (1973). Paradoxical facilitation of object reversal learning after transection of the fornix in monkeys. Neuropsychologia, 11, 271-284.

Zola-Morgan, S., \& SQuire, L. (1990). Neuropsychological investigations of memory and amnesia: Findings from humans and nonhuman primates. In A. Diamond (Ed.), The development and neural bases of higher cognitive functions (Annals of the New York Academy of Sciences, Vol. 608, pp. 434-456). New York: New York Academy of Sciences.

Zola-Morgan, S., SQuire, L., \& Amaral, D. (1986). Human amnesia and the medial temporal region: Enduring memory impairments following a bilateral lesion limited to field $\mathrm{CA} 1$ of the hippocampus. Journal of Neuroscience, 6, 2950-2967.

(Manuscript received August 17, 1999; revision accepted for publication November 3, 1999.) 